\title{
Formulation and characterization of nanoemulgel mangosteen extract in virgin coconut oil for topical formulation
}

\author{
Kamarza Mulia*, Rosalia M.A. Ramadhan, and Elsa A. Krisanti \\ Chemical Engineering Department, Faculty of Engineering, Universitas Indonesia, Depok 16424, Indonesia
}

\begin{abstract}
A hydrogel of nanoemulsion system containing mangosteen extract of mangosteen rind was developed and characterized for the purpose of topical formulation. Mangostins, the bioactive in the extract, has been known to have biological activities such as antifungal, antibacterial, antioxidant, antiviral and antitumoral. Due to the hydrophobic nature of mangostins, they can not to be directly formulated into a gel. Oil-in-water nanoemulsions were formulated of virgin coconut oil (VCO) as the oil phase and mixed surfactant consisting of Tween 80 and Span 80 , using the high-speed homogenization method. Nanoemulgel formed by mixing the nanoemulsion with aqueous solution of xanthan gum, and added phenoxyethanol as a preservative, was a homogeneous milky white gel. The stability test through accelerated centrifugation and freeze-thaw cycle showed that the nanoemulgel would be stable for at least one year. The nanoemulgel penetrated the skin layer up to $12 \mu \mathrm{g} / \mathrm{cm}^{2}$ or more than $95 \%$ of its total mangostin content, better than the skin penetration result of the nanoemulsion formulation. The present study revealed that VCO-mangostins nanoemulgel formulation is a prospective topical formulation.
\end{abstract}

\section{INTRODUCTION}

Cosmetics and skin care products, which composed of numerous chemicals, are recently becoming one of the most-demanded product. Particularly in the skin care category, it has become one of the basic and daily necessities. The products are declared to be safe by the industry as the amount of chemicals added is acceptable. However, regular of these products may result in a chemical accumulation, and hence, increases the chance of unwanted adverse effects. Further development of natural skin care products has to be made in order to reduce the use of harsh chemicals by using the ingredients extracted from plants, such as Garcinia mangostana L., commonly known as mangosteen. Mangosteen is a tropical fruit that grows mainly in Southeast Asia, Southwest India, and other tropical area such as Puerto Rico and Florida. The secondary metabolites, known as xanthones contained in fruit and leaves of mangosteen, possess therapeutic activities such as antioxidant, anti-tumoral, anti-inflammatory, antiallergy, antibacterial, antifungal and antiviral [1]. The major component of xanthones present in mangostana fruit rind is $\alpha$-mangostin, a compound that has been proven to inhibit certain microbes such as Bacillius subtilis and Staphylococcus aureus, which justify the topical uses of G. Mangostana to treat infection in traditional medicine [2].
To achieve effective topical application of mangostins, these bioactives should diffuse through the skin where the diffusion process is dependent on its aqueous solubility, diffusivity, oil or water partition coefficient, concentration in the formulation vehicle, the surface area of the skin to which it is exposed, and the thickness of the stratum corneum according to Fick's Law. Unfortunately, mangostins contained in the extract, which act as antioxidants, can be easily degraded. Encapsulation of mangosteen extract into nanoemulsion can avoid or delay the degradation, as well as to increase the bioavailability of the of mangostins [3].

Nanoemulsions are emulsions made of oil, surfactant, and water, with droplet size in the range of $20-200 \mathrm{~nm}$ [4] or from 50-1000 nm [5]. They can present as oil-in-water $(\mathrm{O} / \mathrm{W})$ emulsions or and water-in-oil $(\mathrm{W} / \mathrm{O})$ emulsions, where the disperse phase of the emulsion is either oil or water, respectively. With its special characteristics, nanoemulsions are ideal vehicles for the purpose of delivery drug or active compounds that lead to enhancement in the therapeutic efficacy by maximizing

\footnotetext{
Corresponding author: kmulia@che.ui.ac.id
} 
the drug penetration into the skin, also minimizing the adverse effect and toxic reactions [6]. Nanoemulsions can easily penetrate the pores of the skin carrying the active substance to the target location, resulting in an effective drug delivery system.

Nanoemulsions are considered to be more stable than conventional emulsions because of the small droplet size that would prevent phase separation. The continuous water phase in the $(\mathrm{O} / \mathrm{W})$ nanoemulsion can increase hydration of the top layer of the skin (stratum corneum), which leads to a rapid increase in the permeability and then a slow down to a steady-state diffusion $[7,8]$. It was also reported that the nano-sized droplets resulted in uniform distribution on the skin, larger surface area, modified release and drug carrier properties, better occlusiveness, film formation on the skin, high stability, and gives pleasant aesthetic character and skin feel [9].

In this study, the mangosteen extract-loaded nanoemulsions were prepared using the high speed homogenization method. The virgin coconut oil (VCO) was selected as the oil phase, where the extract was dissolved within. In addition to having a role as oil phase in $(\mathrm{O} / \mathrm{W})$ nanoemulsions, VCO has many benefits in skin formulas. It can reduce skin moisture, and also improve the skin's moisture-locking barrier. The fatty acids contained also have antimicrobial effects by disrupting bacterial, fungal, and viral cell membranes [10-12]. Tween 80 and Span 80, as non-ionic surfactants, were used in some mole ratios to obtain the proper value of hydrophilic lipophilic balance (HLB) surfactants that form the most stable nanoemulsion.

The low viscosity of nanoemulsion limits its application in transdermal delivery due to its inconvenient use [14]. It was reported that gels are the best formulation as well as better controlled release, absorption properties as well as bioavailability [14]. Incorporating nanoemulsion into gel makes it a dualcontrol release system, which possesses both advantages of nanoemulsions and gels. Apart from better penetration ability, it is also thixotropic, greaseless, non-staining, and easily spreadable, unlike any other topical formula which requires excess rubbing [15]. The gelling agent was selected in this study was xanthan gum.

Xanthan gum consists of a repeating unit of pentasaccharide with glucuronic acid residue between two mannose units as the side chain. Higher degree of viscosity can be obtained from xanthan gum solutions even at low concentrations, unlike the other polysaccharide. Its elastic properties make it a great stabilizer, which allows the suspension of solid particles. Xanthan gum is also very resistant to $\mathrm{pH}$-variations, meaning that it is stable in both alkaline and acidic conditions. Xanthan gum has the best thermal stability compared to other water-soluble hydrocolloids, which provides better stability of the formulation, despite being kept in a refrigerator, stored at room temperature, or heated [16].

The objectives of this study was to obtain the physicochemical properties of nanoemulgel that were formed by using VCO as the oil phase in $\mathrm{O} / \mathrm{W}$ nanoemulsions and xanthan gum as a gelling agent in the water-continuous phase. The $\alpha$-mangostin content, $\mathrm{pH}$, organoleptic, and viscosity were measured within twoweek period. Accelerated stability test of nanoemulgel was performed using centrifugation kecepatan tinggi and freeze-thaw cycle. To study topical application of nanoemulgel, the skin penetration characteristics of nanoemulgel were determined using an in-vitro test using a Franz diffusion cell with abdominal skin of rat as the model skin.

\section{EXPERIMENTAL}

\subsection{Materials}

Mangosteen pericarp was obtained from Solo, central Java, Indonesia, and was identified as G. mangostana L. by Herbarium Bogoriense, Research Center for Biotechnology, Indonesian Institute of Sciences (LIPI). Ethyl acetate, 96\% ethanol, Sodium biphosphate and potassium biphosphate and xanthan gum were bought from Merck, Span 80 and Tween 80 from Sepppic and Virgin Coconut Oil (VCO) were from Kautsarherbal.

\subsection{Extraction of mangosteen pericarp and mangostin quantification}

The maceration using $96 \%$ ethanol for 7 days was done to extract mangostin from the mangosteen fruit pericarp powder, followed by fractionation in a mixture of water ethyl acetate with a ratio of 1:1. The mangostin-rich ethyl acetate fraction was dried in rotary evaporation to obtain dry paste of mangosteen extract. The quantitative analysis of total mangostins content in the extract was carried out by using a UV spectrophotometer and represented as $\alpha$-mangostin content. The absorbance of the extract sample solution and standard solution of $\alpha$ mangostin were measured at a wavelength of $316 \mathrm{~nm}$. A similar procedure was applied for measuring the content of $\alpha$-mangostin in nanoemulsion and nanoemulgel samples.

\subsection{Nanoemulsion and nanoemulgel preparation}

The nanoemulsion was prepared using VCO as oil phase, where the mangosteen extract was dissolved in it. The surfactants used were combination of Tween 80 and Span 80 that gave a HLB value of 12 . Tween 80 was dissolved in the aqueous phase, while Span 80 was dissolved in the oil phase of the VCO. The oil phase was then added into the aqueous phase under stirring, using the high-speed homogenizer, Ultra Turrax T18, for 15 minutes with speed at $10000 \mathrm{rpm}$.

Based on procedure reported by Bhura et al. (2015) [17], with slight modification, hydrogel of nanoemulsion or nanoemulgel was obtained by mixing the prepared nanoemulsion with the gel base in 1 to 1 volume ratio, under gentle stirring. The gel base was prepared by dissolving xanthan gum in distilled water. The amount of xanthan gum added into distilled water was varied into $1.0,1.5$ and $2.0 \%(\mathrm{w} / \mathrm{w})$. Phenoxyethanol was added lastly to the formed nanoemulgel as a preservative. 
Nanoemulgel was characterized visually, and chemically by measuring its $\mathrm{pH}$ and mangostin content retained for two-week period. The composition of nanoemulgel with different weight of gel bases were shown in Table 1.

Table 1. Composition of nanoemulgel with HLB 12 surfactant and xanthan gum gelling agent

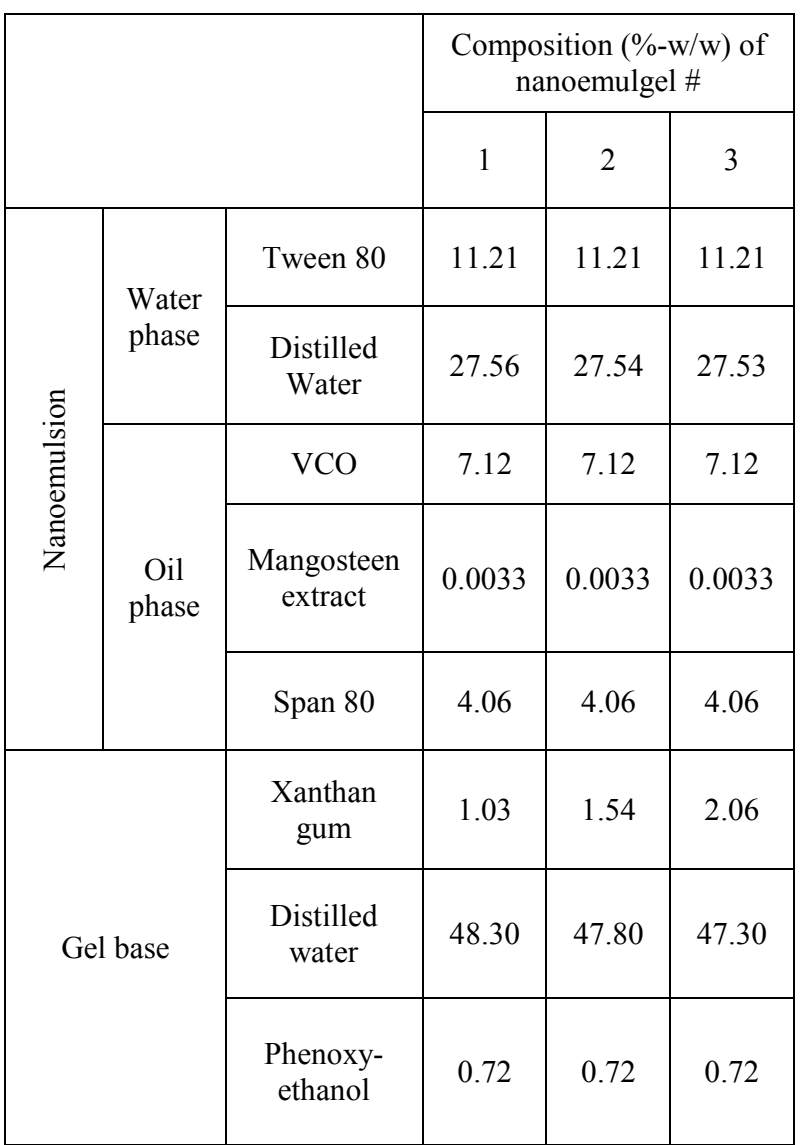

The stability and physicochemical characteristic of nanoemulgel samples were determined by performing several tests, i.e. measurement of viscosity, $\mathrm{pH}$, accelerated stability, alpha mangostin content, organoleptic and customer evaluation.

\subsection{Accelerated stability testing}

To determine the predicted stability of nanoemulgel for long period, there were two tests conducted.

\subsubsection{Centrifugation}

The accelerated stability test was carried out by centrifugation of samples inside a $10-\mathrm{cm}$ centrifuge tube at 3800 RPM for five hours. The stability of samples was observed when there was no phase separation after centrifugation. In his report, Laba (1993) [18] mentioned that centrifugation of sample in this condition was equivalent with the effect of gravity of one year. Thus, all samples that showed no phase separation after centrifugation have shelf life of one year.

\subsubsection{Freeze-Thaw cycle}

Nanoemulgel prepared was also determined its stability using this test as reported by Jadhav et al., (2014) [19]. The samples were stored at $-10{ }^{\circ} \mathrm{C}$ for 48 hours, then removed and allowed to thaw up to $25{ }^{\circ} \mathrm{C}$. The parameters measured for stability were the $\mathrm{pH}$ and $\alpha$ mangostin content for each formulation. The whole steps were repeated for three cycles.

\subsection{Organoleptic tests}

All samples of nanoemulsions and nanoemulgels were observed physically every week for four-week period. The parameters observed were the color, scent and visual phase separation.

\subsection{Viscosity and $\mathrm{pH}$ measurement}

These tests were applied to nanoemulsion samples. The viscosity of nanoemulgel with various content of xanthan gum was measured using Cannon-Fenske capillary viscometer. The stability of nanoemulsion samples was also observed by measuring the $\mathrm{pH}$ every 4 days for two-week period. The changes of $\mathrm{pH}$ during that period of time might indicate instability of nanoemulsion.

\subsection{Mangostins quantity measurement}

Quantitative analysis of the mangostin extract was carried out using a UV spectrophotometer. A stock solution was prepared by diluting $2.5 \mathrm{mg}$ mangostin extract in $25 \mathrm{ml}$ of ethanol, followed by further dilution to obtain a variation of the solution concentration. The absorbance of each solution concentration was measured using a UV spectrophotometer at a wavelength of 316 $\mathrm{nm}$. The total mangosteen or xanthone in the extract can be determined as the amount of $\alpha$-mangostin content measured and calculated based on the standard $\alpha$ mangostin calibration curve that has been prepared.

\subsection{In-vitro skin penetration study}

The skin penetration study was performed by Franz diffusion cell with abdominal skin of rat as the model skin. The sample skin was placed between the donor and the receptor compartment, with stratum corneum side facing the donor. The donor medium consisted of sample formulation, while the receptor was phosphate buffer saline $(\mathrm{pH}$ 7.4). The receptor medium $(0.5 \mathrm{~mL})$ was withdrawn after $0,10,30,60,90,120,180,240$, and $300,360,420,480$ minutes and analyzed by UVspectrophotometer to determine the mangostin content [20].

\subsection{Customer testing}

This test was conducted only for nanoemulgel samples, which was an additional test to help deciding the best formulation by involving consumer acceptance and 
satisfaction. The formulations were evaluated according to its consistency, color, and odor; with consistency and odor being the more significant parameter.

\section{RESULT AND DISCUSSION}

The mangosteen extract in the ethyl acetate fraction was obtained and analyzed using UV-spectrophotometer for its $\alpha$-mangostin content, that represent the amount of total mangostin within the extract. The result showed the total amount of mangostin was $57.2 \%$ (weight) of total dry mangosteen extract. The mangosteen extract of the ethyl acetate fraction was used for preparation of nanoemulsion and nanoemulgel.

\subsection{Mangostin extract in ethyl acetate fraction}

Maceration of dried mangosteen pericarp was conducted for 7 days with periodic stirring which was aimed to increase the contact between simplicia and the solvent. The thickened solvent was fractionated using ethyl acetate and distilled water $(1: 1)$. Phase separation occurred naturally, since ethyl acetate is semi-polar and water is polar solvent. The upper part, which is the ethyl acetate fraction containing mangostins, was then collected and subjected to rotary evaporator to remove the ethyl acetate solvent, leaving the dried paste extract that is rich in mangostins.

\subsection{Nanoemulgel characterization}

The characteristics of nanoemulgel were observed based on the organoleptic, viscosity, $\mathrm{pH}$ determination, mangostin quantitative analysis and stability test.

\subsubsection{Organoleptic, viscosity and $\mathrm{pH}$ determination}

The obtained nanoemulgels were milky-white and homogeneous, with odor comparable to the VCO. The viscosity of the nanoemulgels increases with the increase of xanthan gum concentration within the formulation. Within two-week observation period at room temperature, all of the nanoemulgel formulations with various amounts of xanthan gum remain stable; no phase separation observed, and no changes in consistency or color.

As shown in Fig.1, there were no significant changes of $\mathrm{pH}$ within observation time and no significant effect from variation of $\mathrm{XG}$ concentration. It was found that the $\mathrm{pH}$ measured of nanoemulgel in 12-day observation has minimum $\mathrm{pH}$ value of 5.5 and maximum 6.4 , which is within the skin $\mathrm{pH}$ human skin $(4.5-6.5)$ reported by Leonardi, et al., (2002) and Zlotogorski (1987) [21,22]. The relatively constant value of $\mathrm{pH}$ within two weeks indicated the stability of nanoemulgel.

The $\mathrm{pH}$ measurement was also conducted for all nanoemulgel samples with different amount of xanthan gum (XG) at every four days for two-week observation period.

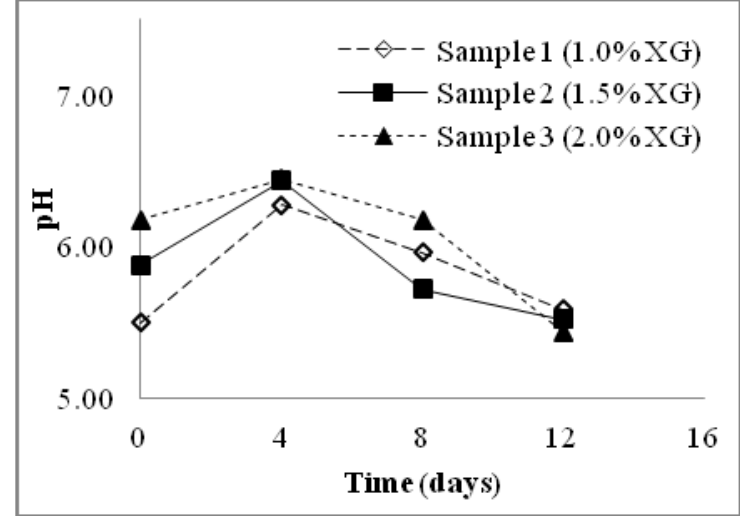

Fig. 1. Plot of $\mathrm{pH}$ of nanoemulgels in two-week period with different ratio of Xanthan Gum (XG) in gel-base aqueous solution.

As shown in Fig.1, there were no significant changes of $\mathrm{pH}$ within observation time and no significant effect from variation of XG concentration. It was found that the $\mathrm{pH}$ measured of nanoemulgel in 12-day observation has minimum $\mathrm{pH}$ value of 5.5 and maximum 6.4 , which is within the skin $\mathrm{pH}$ human skin $(4.5-6.5)$ reported by Leonardi, et al., (2002) and Zlotogorski (1987) [21,22]. The relatively constant value of $\mathrm{pH}$ within two weeks indicated the stability of nanoemulgel.

\subsubsection{Mangostin quantitative analysis}

The quantitative analysis of the mangosteen extract in ethyl acetate fraction was carried out using UV spectrophotometer. The absorbance of the extract sample solution and standard solution of $\alpha$-mangostin were measured at wavelength of $316 \mathrm{~nm}$. The amount of mangosteen in extract of ethyl acetate fraction was measured as $57.9 \%(\mathrm{~g} / \mathrm{g})$.

The $\alpha$-mangostin content of each formulation of nanoemulgel was measured every four days for two week period, and no significant change observed during time of observation, i.e around $0.002 \%(\mathrm{w} / \mathrm{w})$. This experimental data was slightly less than the calculated total mangosteen extract $(0.0033 \%)$ in the nanoemulgel, as shown in Table 1.

The observation of $\alpha$-mangostin and $\mathrm{pH}$ in two weeks showed that both $\mathrm{pH}$ and $\alpha$-mangostin were stable. This stability was attained due to the chemical structure of xanthan gum as the gelling agent. The glucan backbone is protected by the side chains which lie alongside, making it relatively stable to acids, alkalis and enzymes, factors that may trigger molecules collisions and hence degradation [23]. Regardless of the value of $\alpha$-mangostin content that were quite small in nanoemulgel, the results showed that the variation of $\mathrm{XG}$ content in the gel base showed no effect on the content of mangostin within the nanoemulgel as well as on the $\mathrm{pH}$. 


\subsubsection{Accelerated stability testing for shelf life}

All nanoemulgel samples were centrifuged at 3800 RPM and after five hours there were no phase separation observed. This result indicates the stability of all hydrogel formulations that equals to having a shelf life of one year. The stability of nanoemulgel using these formulations might be due to the presence of xanthan gum that reduce the interfacial tension and tends to form a cohesive interfacial film around the nanoemulsion droplets, hence improving the stability of nanoemulgel [24]. The effect of xanthan gum also can be explained by its ability, as hydrocolloid, to stabilize and slow the thermodynamically breakdown of gel [23].

\subsubsection{Freeze-Thaw cycle test for stability detection of nanoemulgel}

All of the formulations were also subjected to freezethaw testing where the samples are subjected to two extreme temperature changes, which in this case is $-10^{\circ} \mathrm{C}$ and $25^{\circ} \mathrm{C}$. There was no sign of phase separation for any formulations, indicating that the nanoemulgel would be stable even under such condition, which could likely be encountered in real life. $\alpha$-Mangostin content in all formulations that have been subjected to this test, were evaluated using UV-spectrophotometer with similar procedure described previously. The $\alpha$-mangostin analysis and $\mathrm{pH}$ measurement after conduction of Freeze-Thaw test are reported in Table 2.

Table 2. The $\mathrm{pH}$ and mangostin content of various nanoemulgel formulations as a function of freeze-thaw cycles.

\begin{tabular}{|c|c|c|c|c|}
\hline \multicolumn{2}{|c|}{} & \multicolumn{3}{|c|}{$\begin{array}{c}\text { Nanoemulgel \# } \\
(\%-\mathrm{w} / \mathrm{w} \text { XG) }\end{array}$} \\
\hline \multirow{4}{*}{ Parameter } & Cycle & $\begin{array}{c}1 \\
(1.0 \%)\end{array}$ & $\begin{array}{c}2 \\
(1.5 \%)\end{array}$ & $\begin{array}{c}3 \\
(2.0 \%)\end{array}$ \\
\hline \multirow{4}{*}{$\mathrm{pH}$} & 0 & 5.70 & 5.48 & 5.28 \\
\cline { 2 - 5 } & 1 & 5.65 & 5.58 & 5.71 \\
\cline { 2 - 5 } & 2 & 6.21 & 6.10 & 6.20 \\
\cline { 2 - 5 } & 3 & 6.58 & 6.53 & 6.52 \\
\hline \multirow{4}{*}{$\begin{array}{c}\alpha-m a n g o s t i n \\
\text { content (\%) }\end{array}$} & 1 & 0.0018 & 0.0019 & 0.0018 \\
\cline { 2 - 5 } & 2 & 0.0018 & 0.0018 & 0.0018 \\
\cline { 2 - 5 } & 3 & 0.0017 & 0.0018 & 0.0018 \\
\hline
\end{tabular}

Note: $X G=x a n t h a n$ gum, the composition of samples \# 1,2 and 3 are described in Table 1. Each cycle is performed at $-10^{\circ} \mathrm{C}$ and $25^{\circ} \mathrm{C}$.

It is shown in Table 2, the content of $\alpha$-mangostin in the nanoemulgel in all samples were relatively constant, around $0.0018 \%$, regardless of the differences in xanthan gum amount. The value in three cycles was not very far from the initial concentration of $\alpha$-mangostin in the emulgel, which was $0.002 \%$. The result indicated that even as low as $1 \%$ XG has given effect on stability of nanoemulgel. The stability of nanoemulgel in these formulations might be due to the good thermal stability of xanthan gum, having a rigid helical conformation that may provide better stability despite being kept in a refrigerator [16].

As the nanoemulgel samples frozen, numerous small ice crystals would be generated and the size may increase as the samples cycled through freeze/thaw temperatures. Kuntz (1995) [25] reported that the presence of xanthan gum within the nanoemulgel samples may help in controlling crystal growth by binding the water, keeping it from coalescing into bigger ice crystals. Xanthan gum is also attributed to possible steric blocking of the oil droplet interfaces or inhibiting solute transport at the interface by forming hydrocolloids gel [26].

The $\mathrm{pH}$ of each composition was also measured at the end of each cycle and showed an increase as shown in Table 2 and Fig 2. This is due to the reason that two phases would be generated at the end of freezing process; a region with pure ice crystals, and the unfrozen solutes are effectively crowded into a smaller remaining liquid volume. The effective concentration of unfrozen solutes will increase with the number of cycles, that shift the $\mathrm{pH}$ of the formulation [27]. Nevertheless, the $\mathrm{pH}$ range measured after the cycles was still within the acceptable $\mathrm{pH}$ range (4.5-6.5).

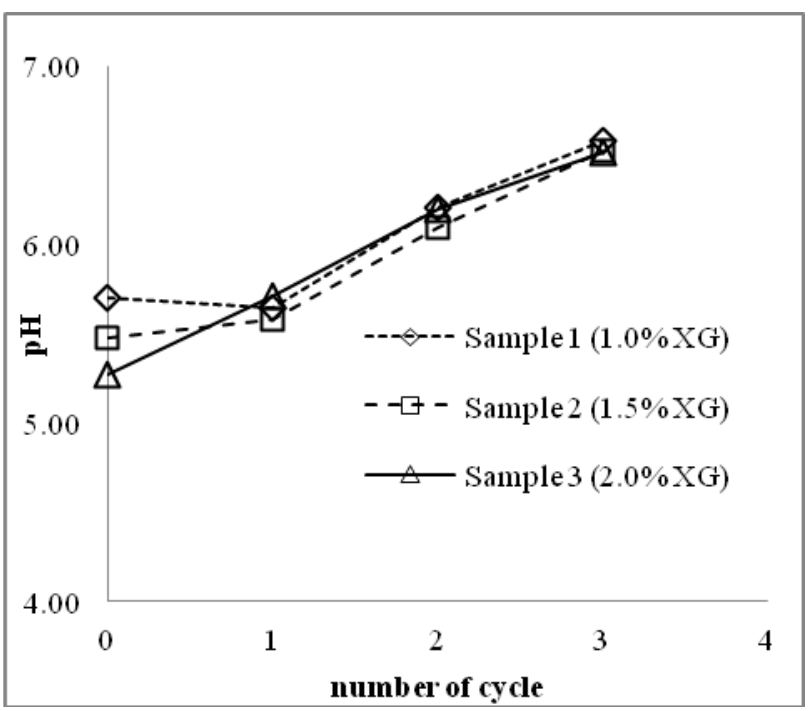

Fig. 2. The pH of nanoemulgels at 3 cycles of freeze-thaw testing with different ratios of xanthan gum in the gel-base aqueous solution

\subsection{In-vitro skin penetration study of nanoemulgel}

The advantage of delivering active compounds through nanoemulgel was mentioned by Basera, et al. (2015) [28], due to better adhesion to the surface of the skin and the high solubilizing capacity, which leads to larger 
concentration gradient towards the skin, and hence better skin penetration. Chellapa et al. (2015) [29] also reported that upon skin contact nanoemulgel will release the oily droplets from the gel network that then penetrate into the stratum corneum of the skin and directly deliver the drug or active molecules.

The ability of different formulations of the gel to penetrate the skin was studied. At the same time, nanoemulsion was also subjected to the same test as a comparison, as seen in Fig 3. In the first ten minutes the cumulative amount of penetrating mangostin reached about $6 \mu \mathrm{g} / \mathrm{cm}^{2}$ or $50 \%$ of initial content and it increased slowly to about $12 \mu \mathrm{g} / \mathrm{cm}^{2}$ or $95 \%$ at about eight hours. The addition of xanthan gum to the nanoemulsion around 1-1.5 \% (w) significantly increased the skin penetration of the mangostin extract, however the addition of more xantham gum to $2 \%$ (w) lower the penetration. It seems that $1.5 \%(\mathrm{w})$ is the optimum amount of xanthan gum in the nanoemulgel, that showed the highest skin penetration of mangostin, while nanoemulsion without xanthan gum showed the lowest.

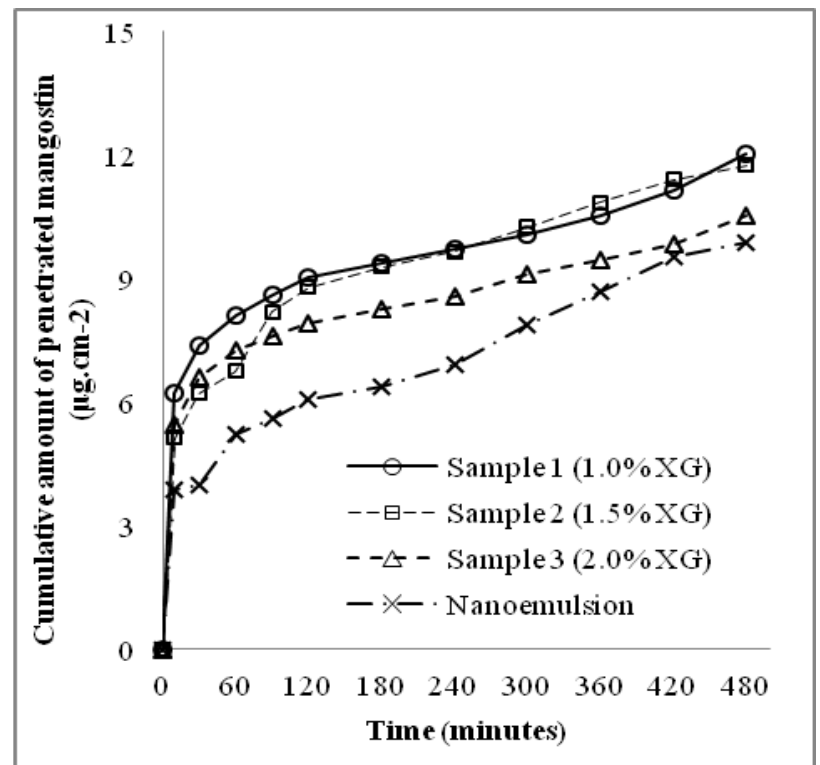

Fig. 3. Penetration of mangostin through skin membrane: Cumulative penetrated mangostin $\left(\mu \mathrm{g} / \mathrm{cm}^{2}\right)$ of nanoemulgel and nanoemulsion samples with xantham gum.

Thermodinamic activity of active compound or drug in the applied solution, such as nanoemulgel, affect the penetration of drug through a skin membrane [30], as given in the steady-state Fick's 1st law of diffusion of a drug through skin $(\mathrm{J})$ :

$$
l=\frac{A_{W} D}{Y_{s} L}
$$

where $A_{v}, D, \gamma_{s}$ and $L$ are the thermodinamic activity of the drug in a formulation, the diffusion coeffient, the activity coefficient of the drug in the skin barrier, and the thickness of the skin barrier, respectively. Salim, et al. (2012) [31] reported that addition of hydrocolloid gum, such as xanthan gum, increased the lipophilicity of the oil droplets, and thus increased the thermodynamic activity of the drug, that lead to the increase of the skin penetration rate. This study showed similar results, i.e. the addition of xanthan gum to the nanoemulgel increased the flux of mangostin that penetrated the skin membrane.

\subsection{Customer testing for nanoemulgel}

Customer testing was conducted as an additional test to help deciding the best formulation by involving consumer acceptance and satisfaction. The formulations were evaluated according to some parameters, i.e. consistency, color, and odor. Each parameter has different weight according to the level of importance, affecting customer acceptance of the sample product. Consistency and odor are more significant parameter compared to color, so they have a weight of $40 \%$ each, while the color has a weight of $20 \%$. This is due to the non-staining and soft color of the nanoemulgel, and hence, the less effect of color on the customer acceptance. Most of the respondents were satisfied with the consistency, color, and odor of emulgel formulation number 1 with $1 \%$ of xanthan gum. It is then concluded that the addition $1 \%$ of xanthan gum into the formulation will produce the most suitable gel consistency, odor as well as maintain the stability.

\section{CONCLUSIONS}

A prospective mangostin nanoemulgel for topical formulation using virgin coconut oil as oil phase in making nanoemulsion, and xanthan gum as a gelling agent in aqueous solution, was developed and characterized. Milky-white, homogeneous nanoemulgels were obtained from mixing the gel base (xanthan gum solution), and nanoemulsion with 1:1 weight ratio. The results of accelerated test showed that the stability of all nanoemulgel formulations equals to having a shelf life of one year. The Freeze-Thaw cycle test indicated that as low as $1 \%(w)$, xanthan gum has given significant effect on stability of nanoemulgel.

Nanoemulgels were also confirmed to afford better penetration ability than its nanoemulsion $\left(12 \mu \mathrm{g} / \mathrm{cm}^{2}\right.$ or $95 \%$ cumulative). The skin penetration test showed that addition of xanthan gum to the nanoemulsion as much as $1 \%$ (w) significantly increased the skin penetration of mangostins, however, the $1.5 \%(\mathrm{w})$ is the optimum amount of xanthan gum in the nanoemulgel.

When nanoemulsion was combined with an aqueous gel based to form nanoemulgel, the results showed that the variation of xanthan gum content in the gel base showed no effect on the content of mangostin as well as on the $\mathrm{pH}$ of nanoemulgel. Based on customer testing, the nanoemulgel containing $1 \%$ of xanthan gum was the most accepted by the respondents.

\section{ACKNOWLEDGEMENT}

The authors are grateful for financial support from the Universitas Indonesia through the DRPM PITTA Grant 2017. 


\section{REFERENCES}

1 J. Pedraza-Chaverri, N. Cárdenas-Rodríguez, M. Orozco-Ibarra, and J.M. Pérez-Rojas, Food Chem. Toxicol. 46, 3227 (2008).

2 S. Al-Massarani, A. El Gamal, N. Al-Musayeib, R. Mothana, O. Basudan, A. Al-Rehaily, M. Farag, M. Assaf, K. El Tahir, and L. Maes, Molecules 18, 10599 (2013).

3 K.O. Aboalnaja, S. Yaghmoor, T.A. Kumosani, D.J. McClements, Expert Opinion on Drug Delivery. 13, $1327,2016$.

4 C. Solans, P. Izquierdo, J. Nolla, N. Azemar, And M. Garciacelma, Curr. Opin. Colloid Interface Sci. 10, 102 (2005).

5 C. Lovelyn and A.A. Attama, J. Biomater. Nanobiotechnol. 2, 626 (2011).

6 M. Jaiswal, R. Dudhe, and P.K. Sharma, 3 Biotech 5, 123 (2015).

7 E. Abd, Targeted Skin Delivery of Topically Applied Drugs by Optimized Formula Design, University of Queensland, 2015.

8 A. Vyas, A.K. Sonker, B. Gidwani, The Scientific World Journal, Article ID 276260, 2014.

9 M.N. Yukuyama, D.D.M. Ghisleni, T.J.A. Pinto, and N.A. Bou-Chacra, Int. J. Cosmet. Sci. 38, 13 (2016).

10 G. Bergsson, J. Arnfinnsson, O. Steingrimsson, and H. Thormar, Antimicrob. Agents Chemother. 45, 3209 (2001).

11 S. Intahphuak, P. Khonsung, and A. Panthong, Pharm. Biol. 48, 151 (2010).R. Arora, G. Aggarwal, S.L. Harikumar, and K. Kaur, Adv. Pharm. 2014, 1 (2014).

12 M. Shilling, L. Matt, E. Rubin, M.P. Visitacion, N.A. Haller, S.F. Grey, and C.J. Woolverton, J. Med. Food 16, 1079 (2013).

13 R. Arora, G. Aggarwal, S.L. Harikumar, and K. Kaur, Adv. Pharm. 2014, 1 (2014).

14 L.P. Kaur and T.K. Guleri, Asian J. Biomed. Pharm. Sci. 3, 1 (2013).

15 R. Wani, M. Patil, P. Dhurjad, C. Chaudhari, and S. Kshirsagar, Int. J. Pharm. Res. Sch. 4, 397 (2015).

16 C. Qiu, M. Zhao, D. J. McClements, Food Hydrocolloids, 43, 377 (2015).

17 M.R.G. Bhura, K.A. Bhagat, and S.K. Shah, World J. Pharm. Sci. 3, 1013 (2015).

18 D. Laba, Rheological Properties of Cosmetics and Toiletries (CRC Press, 1993).

19 C. Jadhav, V. Kate, and S.A. Payghan, J. Nanostructure Chem. 5, 107 (2015).

20 P. Widyanati, M. Jufri, and B. Elya, Int. J. Pharm. Sci. Rev. Res 27, 1 (2014).

21 G.R. Leonardi, L.R. Gaspar, and P.M.B.G. Maia Campos, An. Bras. Dermatol. 77, 563 (2002).

22 A. Zlotogorski, Arch. Dermatol. Res. 279, 398 (1987).
23 M. Chaplin, Xanthan Gum (2016).

24 S.H. Saharudin, Z. Ahmad, and M. Basri, Int. Food Res. J. 23, 1361 (2016).

25 L.A. Kuntz, Freeze/Thaw-Stability (1995).

26 B.M. Degner, C. Chung, V. Schlegel, R. Hutkins, and D.J. McClements, Compr. Rev. Food Sci. Food Saf. 13, 98 (2014).

27 J. Brunstein, Med. Lab. Obs. 44 (2015).

28 K. Basera, G. Bhatt, P. Kothiyal, and P. Gupta, World J. Pharm. Pharm. Sci. 4, 1871 (2015).

29 P. Chellapa, A.T. Mohamed, E.I. Keleb, A.M. Eid, Y.S. Issa, and N.A. Elmarzugi, IOSR J. Pharm. 5, 43 (2015).

30 H. Ishii, H. Todo, and K. Sugibayashi, Chem. Pharm. Bull. (Tokyo). 58, 556 (2010).

31 N. Salim, M. Basri, M.B. Rahman, D.K. Abdullah, H. Basri, Int. J. Nanomedicine, 7, 4739 (2012). 